\title{
Specialized CNT-based Sensor Framework for Advanced Motion Tracking
}

\author{
Federico Gelsomini ${ }^{\mathbf{1}, 4}$, Patrick C. K, Hung ${ }^{\mathbf{2 , 4}}$, Bill Kapralos ${ }^{\mathbf{2 , 4}}$, Alvaro Uribe-Quevedo ${ }^{2}$, \\ Michael Jenkin ${ }^{3,4}$, Akira Tokuhiro², Kamen Kanev ${ }^{2,4}$, Makoto Hosoda ${ }^{4}$, Hidenori Mimura ${ }^{4}$ \\ ${ }^{1}$ Sapienza University of Rome, Rome, Italy \\ ${ }^{2}$ Ontario Tech University, Oshawa, ON, Canada \\ ${ }^{3}$ York University, North York, ON, Canada \\ ${ }^{4}$ Shizuoka University, Hamamatsu, Japan
}

federico.gelsomini@uniromal.it, \{patrick.hung, bill.kapralos, alvaro.quevedo, akira.tokuhiro\}@uoit.ca, jenkin@cse.yorku.ca,kanev@inf.shizuoka.ac.jp, \{hosoda.makoto, mimura.hidenori\}@shizuoka.ac.jp

\begin{abstract}
In this work, we discuss the design and development of an advanced framework for high-fidelity finger motion tracking based on Specialized Carbon Nanotube (CNT) stretchable sensors developed at our research facilities. Earlier versions of the CNT sensors have been employed in the high-fidelity finger motion tracking Data Glove commercialized by Yamaha, Japan. The framework presented in this paper encompasses our continuing research and development of more advanced CNT-based sensors and the implementation of novel high-fidelity motion tracking products based on them. The CNT sensor production and communication framework components are considered in detail and wireless motion tracking experiments with the developed hardware and software components integrated with the Yamaha Data Glove are reported.
\end{abstract}

\section{Introduction}

Experimental tracking and digitization of hand and arm motions in research, education, and training environments are common and their adoption in various practical application areas continues to expand [1, 2]. Since the late 1970s when the first hand tracking gloves were reported [3], motion tracking and digitization technologies have progressed significantly. Nowadays, most smartphones have embedded motion tracking sensors enabling direct gait tracking and hand gesture interactions $[4,5]$. Various types of compact wearable motion trackers are also available from companies such as Mbientlab (https://mbientlab.com/), Xsens (https://www.xsens.com/), and other producers worldwide. Following this trend, motion tracking and analysis are rapidly expanding to the Internet of Things (IoT), addressing applications in sports, health monitoring, medical rehabilitation, amongst many others [6].
The situation is very different, however, when we consider the tracking of the human hand with all of its fingers. Handheld (e.g., smartphones) and wrist-based (e.g., wristbands) tracking devices usually provide only a synthesized six degrees-of-freedom (DOF) position and orientation feedback. Although modern tracking devices may employ a combination of tracking sensors such as accelerometers, gyroscopes, and magnetometers, their combined feedback is typically limited to nine DOF [7]. The full tracking of the five fingers of the human hand, in contrast, requires over 18 DOF which makes the task much more complex. Practical complications also arise since attaching physical tracking devises to all fingers severely impedes the subtle finger motions.

Instead of using tracking devices physically attached to the fingers, finger tracking experiments have been conducted by attaching markers to the fingers and tracking the fingers optically using a small camera mounted on the palm [8]. The resulting system allowed the gathering of research data but was of limited practical use mainly due to its complexity and the burden imposed on the user by the size of the markers and the presence of the camera. In other research projects, the classic motion tracking approach based on spherical retro-reflective markers was used [9]. Although small, the retro-reflective markers had to be attached to all of the fingers, hampering natural finger movements. Experiments with finger tracking of piano players were conducted by employing painted markers that were less invasive [10]. However, the optical tracking method in this system was not self-sufficient as additional capacitive and Musical Instrument Digital Interface (MIDI) sensors were attached to the piano keys. Furthermore, experiments with optical tracking using the Microsoft Kinect and the Leap Motion reported in [11], revealed high latencies and numerous false positives/negatives during piano playing. As a

URI: https://hdl.handle.net/10125/70843

978-0-9981331-4-0

(CC BY-NC-ND 4.0) 
result, an alternative ultrasound-based tracking approach was deemed more robust.

Given the difficulties with optical finger motion tracking, more recent systems have shifted to other, nonoptical methods for direct sensing of finger motions. Such systems are often designed as wired gloves with embedded sensors for direct tracking of the angular positions of the finger joints. Employing wired gloves for finger motion tracking is particularly suitable for applications where gloves are worn as a code of practice. Wired gloves can be seamlessly adopted, for example, in medical training where surgical gloves are an indispensable component of many medical procedures. Of course, such wired gloves should mimic the physical properties of the surgical gloves and provide the trainees with a touch and feel similar to real surgical gloves.

Technological advancements and novel materials, specifically designed to meet the requirements of the target application domain become, therefore, indispensable in wired glove designs. Along with the use of special materials, finger motion tracking sensors that blend into the glove fabric and allow for seamless integration into the wired glove are also required.

The early wired glove designs [3] employed embedded optical sensors constructed from a light emitter and a photocell connected by a flexible rubber tube that bends following the motions of the human fingers [12]. In such sensors, the light reaching the photocell decreases proportionally to the tube bend which is reflected in the output voltage of the photocell. In a similar manner, more performant and easy to handle sensors have been constructed from an optical fiber with an intentionally damaged surface that attenuates the light propagation proportionally to the bend [13]. More advanced fiber-optic sensors based on double cladding fiber have also been reported [14]. Such fiber optic sensors used to be employed in high-end wired gloves, (e.g., the Virtual Programming Languages (VPL) DataGlove [15]), while lower-cost capacitive [16], and resistive bend sensors were commonly used in consumer-grade products (e.g., the Nintendo Power Glove [17]). Some bendable resistive sensors for wired gloves are produced by applying conductive inks to a flexible substrate [18]. However, such sensors are more sensitive to expansion than to compression and thus generate an asymmetric response.

For high fidelity finger motion tracking and the detection of subtle finger movements, sensing of the bending motion is too restrictive and may not provide sufficient information. In such cases, stretchable sensors are deemed more appropriate, and active research is continuing in this field. Currently, stretchable sensors are built employing either capacitive [19, 20, 21], or resistive $[22,23,24]$ technologies. Following this line of research, our team is developing rapid-response, widely stretchable sensors based on carbon nanotubes specialized for the detection of human motions $[25,26$, 27].

\section{CNT sensor production framework}

The specialized stretchable sensors stem from the synthesis of vertically aligned ultralong multi-walled Carbon Nanotubes (CNTs) using iron chloride powder. This unique process, co-invented by one of the authors of the present work [25], is essential for the reliable and cost-effective fabrication of the CNT material embedded in the high-fidelity motion tracking sensors as discussed below.

Initially, a $2.1 \mathrm{~mm}$ long bulk CNT array is grown by conventional thermal chemical vapor deposition on a quartz surface with a single gas flow of acetylene for 20 min. The grown CNT is then spun into a yarn which essentially transforms the three-dimensional array into a horizontally aligned web representing a twodimensional network of CNTs. As a result, CNT webs can be efficiently assembled into large-scale structures without twisting.

Afterward, well-aligned CNT sheets are fabricated by stacking and shrinking the CNT webs [26]. We have produced such a CNT sheet by winding, stacking on a drum, and cutting for proper flattening. The typical number of CNT web layers wound on the drum is between eight and 12 .

Experimental CNT strain sensors are then manufactured by placing the CNT sheet on a flat and smooth substrate (e.g., glass) in a direction parallel to the stretching direction and impregnating it with elastomeric resin. Rubber-like elastomeric resin with low elasticity and low-loss properties is required for this process. After a careful investigation, we selected Polycarbonate-urethane (PCU) resin for its ease of bonding, durability, and hydrolysis and chemical resistance. To enhance the contraction properties of the substrate, we have also employed segmented Polytetramethylene ether Glycol-urethane (PTMGU) [27]. The expansion-contraction capabilities of our CNT sensors are illustrated in Figure 1.

The fabrication of high-fidelity CNT strain sensors requires precise alignment of the CNT bundle direction with the CNT sheet and the electrode directions. Once this is confirmed, the multi-layer structure is combined with the elastomer resin layer. A spin coater has been used to apply the elastomer resin uniformly over the surface of the CNT bundle to a thickness of several dozens of micrometers. 


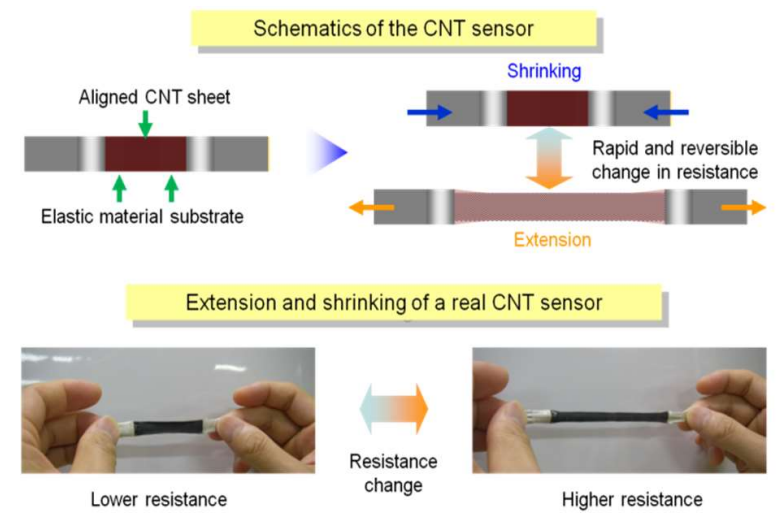

Figure 1. Expansion-contraction properties of the stretchable CNT sensors.

To further stabilize the contraction behavior of the sensor, an elasticity-assist layer is applied on top of the elastomer resin surrounding the CNT bundle. The elastic modulus of the elasticity-assist layer is approximately $2.5 \mathrm{MPa}$ with a $100 \%$ modulus. While the resistance naturally increases with strain due to the cracking of the CNT sheet network, it can be adjusted by using CNT sheets with a different number of layers. In this way, we can produce stretchable sensors with prescribed resistance that allows for more accurate motion tracking.

The sensitivity Gauge Factor (GF) value of a stretchable sensor is defined as $\mathrm{GF}=\left(\Delta \mathrm{R} / \mathrm{R}_{0}\right) /\left(\Delta \mathrm{L} / \mathrm{L}_{0}\right)$, where $R_{0}$ represents the initial resistance, $\Delta R$ is the variation of the resistance, $L_{0}$ represents the initial length, $\Delta \mathrm{L}$ is the variation between the initial length and the elongated length, and $\left(\Delta \mathrm{L} / \mathrm{L}_{0}\right)$ is the strain. The GF value of our CNT-based stretchable sensors obtained via linear approximation is approximately 10.5, indicating a high sensitivity. The sensor resistance is proportional to the applied tensile strain and more specifically, the higher the tensile strain, the higher the sensor resistance. The detection limit of our sensors becomes over 200\% of the applied strain. Furthermore, the strain sensor resistance variation closely follows the temporal strain changes. A series of resistance measurements involving extensions and contractions at different frequencies have been conducted to confirm the excellent rapid response of the sensors [27].

Our sensors also expose a high-linearity of the resistance variation relative to the strain which is particularly important for precise motion tracking. They allow the behavior of the human skin with respect to the finger motions, to be mimicked, and are suitable for both unobtrusive direct attachment to the fingers and integration in different types of gloves. Earlier versions of our specialized stretchable CNT-based sensors have been commercialized and are currently employed in Yamaha Data Gloves [27].

\section{Communications framework}

Our collaborative research with Yamaha stems from the need for high fidelity motion tracking during the play of different musical instruments. The real-time motion tracking of the fingers of a piano player, for example, is a complex task that requires both the employment of advanced sensing technologies and high-performance data processing. Such motion tracking has to be implemented with a minimal burden to the artist so that the free natural movements of the hands and the fingers of the player over the piano keyboard are unimpeded.

The specialized stretchable CNT-based sensors being developed by our team are particularly suitable for this task as they expose properties very similar to the human skin. We are aiming at the fabrication of stretchable sensors that could be directly attached to the fingers of the piano player. Such sensors should track the individual finger motions and communicate the measured data wirelessly.

The framework that we discuss in this paper aims to tackle both, the existing and the forthcoming models of stretchable CNT-based sensors along with the products they are embedded in. The Yamaha Data Glove, being the first commercial product based on our stretchable CNT-sensors, is therefore one of the first framework components that we should elaborate upon.

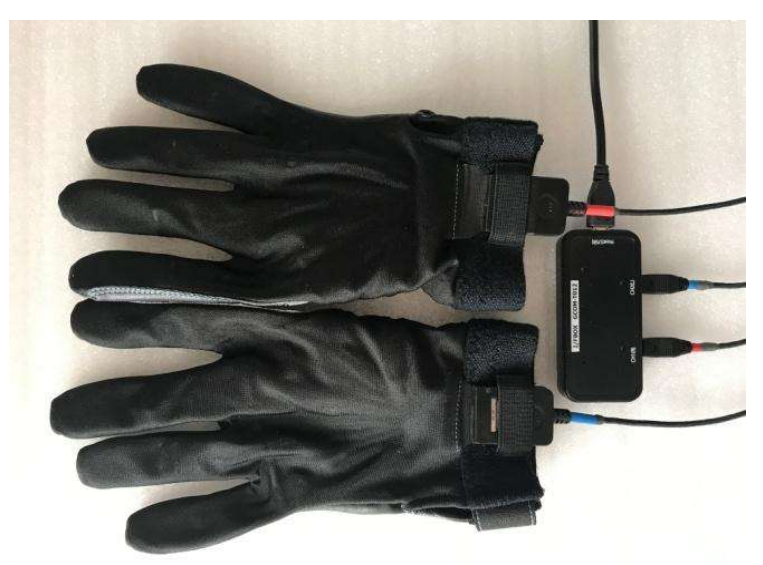

Figure 2. A pair of Yamaha Data Gloves wired to a 2channel USB communication device.

The current Yamaha Data Glove (see Figure 2), must be wired to a controlling computer. This is carried out through a standard Universal Serial Bus (USB) connection that is available on most desktop and laptop computers. The Data Glove set includes a standard Data 
Glove test application (see Figure 3), and demo software for Windows although exemplary source code and/or a Software Development Kit (SDK) are currently not available.

Modern devices include advanced wireless communication capabilities that are well standardized and easy to program across different platforms and OSs. Most smartphones, for example, can communicate using cellular networks (3G, LTE, 5G), WiFi, and Bluetooth. Most of the mobile robots are also enabled for $\mathrm{WiFi}$ and Bluetooth communications [28]. Given the above considerations, it becomes clear that enabling the Data Glove for wireless communications will open a range of new possibilities for use in different applications where wired connections are deemed not suitable. Our framework should, therefore, provide adequate support for the respective wireless communication of the stretchable sensors data.

One of the most obvious options to consider is the employment of a wearable computer with an embedded USB port and OS that allows its wired connection to the Data Glove. With a Windows OS, the existing application software can be used with minimal, if any changes. With this approach, the essential data processing can be performed on the wearable computer which can run the Data Glove related software along with other necessary applications. Obviously, the wearable computing device can also connect wirelessly to the Cloud and/or to other local computing and data processing nodes.

Another possible approach would be to develop a minimalistic device (e.g., a WiFi dongle) wired to the Data Glove capable of retransmitting all communication packets wirelessly to a server computer. This approach will provide no wearable computing facilities so all the data processing must be performed on the server computer which will also run the related user applications. Note that the server computer may be wirelessly connected to a desktop computer, a laptop, a tablet, or a smartphone. This approach allows for easy migration of the data processing software and the user applications between different computers and platforms without any hardware changes. The wireless dongle can, for example, be paired with a powerful workstation on which the software development and the experimental data processing are carried out. For demonstrations and outdoor experiments, on the other hand, the Data Glove can be paired with a mobile device such as a smartphone.

In the following sections, we continue with a more detailed presentation of the wearable computer approach and discuss the essential hardware and software components that we have developed.

\section{Wireless motion tracking experiments}

An ad-hoc wireless version of the Data Glove was built with a Stick Personal Computer (PC). The Data Glove was connected via a USB dongle to the Stick PC's USB port. The dongle is supported by a Silicon Labs USB to the Universal Asynchronous Receiver Transmitter (UART) Bridge that creates a serial COM port and makes it accessible to the software running on the Stick PC. In this way, the standard Data Glove test application can be run on the Stick PC as shown in Figure 3. While this is convenient for verification of the proper driver installation and the Data Glove connections, the Stick PC has a limited computational capacity, so most of the real data processing and experimental calculations should be done on a more powerful workstation.

The Data Glove serial communication protocol is based on 40 byte data packets sent at regular, $4 \mathrm{~ms}$ intervals. The serial channel communication speed is fixed at 460,800 bps, which allows for approximately 3 $\mathrm{ms}$ idle time between the data packets. For reliable tracking and recognition of the finger motions and the hand gestures, timely arrival and processing of the data packets need to be ensured. With respect to this, we have conducted experiments to verify the performance of the employed components and evaluate the jitter of the communication channels.

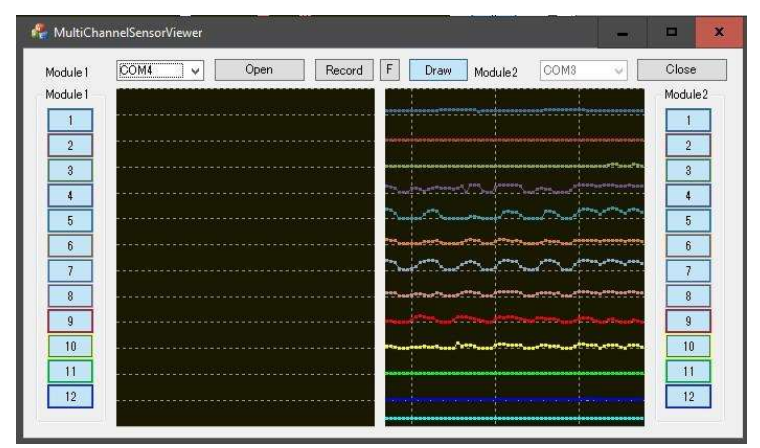

Figure 3. The standard Data Glove test application running on the StickPC with the right hand Data Glove connected.

The performance of the Stick PC (Intel Atom x5z8350 CPU@1.44 GHz; 4 GB RAM), was measured by receiving and decoding a continuous packet stream from a Data Glove connected to one of its USB ports via a DG2USB convertor. Subsequences of 250 packets (each approximately $1 \mathrm{sec}$ long) were extracted from the data stream at random intervals and stored for consequent analysis. All packets in the sequences were timestamped with their arrival times. The timestamps were used for calculating the time intervals $\Delta \mathrm{T}$ between the 
arriving packages. Since the Data Glove generates equally sized packets at a constant rate, the distribution of the $\Delta \mathrm{T}$ values shown on the histogram in Figure 4 can be used as a direct estimate of the jitter. The variance of the $\Delta \mathrm{T}$ values shown in the histogram is approx. $8 \%$ which corresponds to a fairly low jitter.

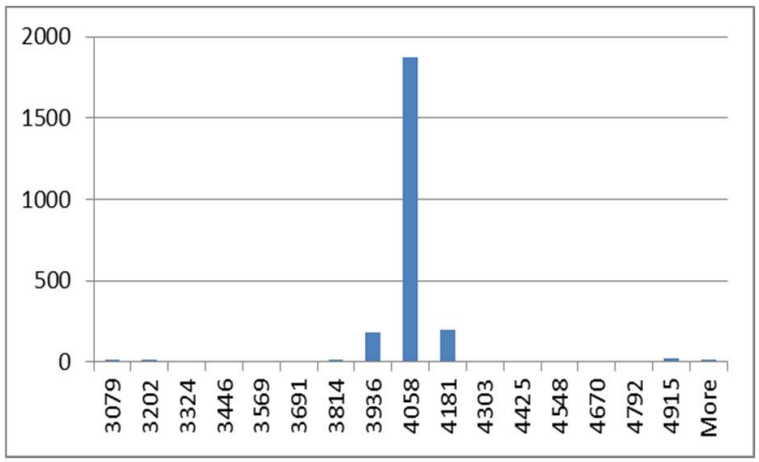

Figure 4. Histogram of the arriving Data Glove packet time intervals $\Delta \mathrm{T}$ in microseconds.

During the experiments, the CPU utilization of the Stick PC was at approx. 37\%, confirming its capacity for stable reception and timely processing of the incoming Data Glove packets. For verification, the same experiment was conducted on a more powerful desktop PC (Intel 17-3770 CPU@3.4 GHz, 8 GB RAM). In this case, the $\Delta \mathrm{T}$ variance was practically the same while the CPU utilization of the desktop PC was less than $15 \%$.

We have configured the Stick PC to automatically connect to the local WiFi network shared with the development workstations in our labs. In this way, the lab computers can run, for example, any Data Glove application by connecting to the Data Glove wirelessly via the Stick PC. For this, we have developed a com2ip (COM port to Internet port) application that runs on the Stick PC. The application creates a server port on the Stick PC that is available for establishing connections from any client that has access to the Internet Protocol (IP) address of the Stick PC.

On the client side, we require an application that connects to the Data Glove server port and establishes a channel to a local COM port that can be used by the other applications. The functionality of the desired application is essentially an inverse of the functionality of the com2ip application. We have, therefore, created an ip2com (Internet port to COM port) application that can be run on any server or a workstation that requires wireless communications with the Data Glove. The employed hardware, drivers, and software components along with their interconnections are schematically depicted in Figure 5.

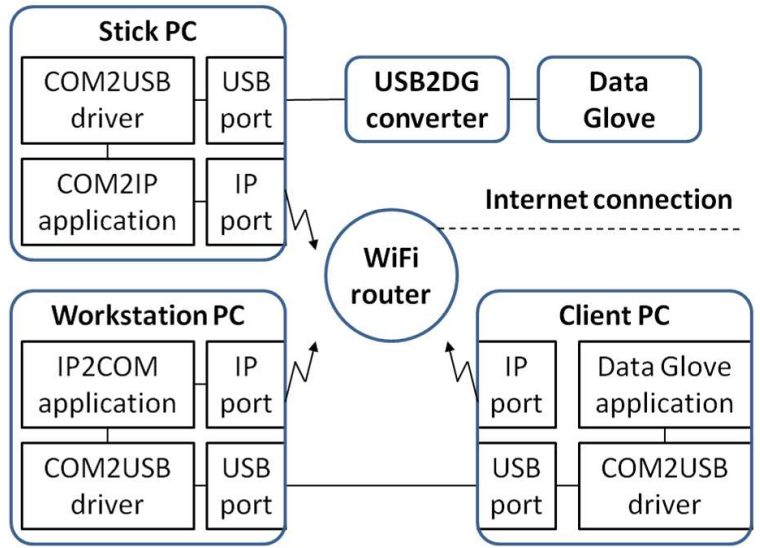

Figure 5. A simplified diagram of the interconnected

Data Glove and related hardware, drivers, and application software components.

Although this is a purely technical issue, we would like to point out that the COM ports for the ip2com application, the standard Data Glove test application, and the user applications might be either physical or virtual. To verify this functionality, we have conducted experiments with physical COM ports by employing two USB2RS232 convertors wired together as shown in Figure 6. We used the convertors to establish an RS232 serial connection between a Workstation and a Client $\mathrm{PC}$ as shown in Figure 5. The ip2com and the Data Glove applications successfully communicated with the Data Glove through the established serial channel at 460,800 bps with fairly low jitter.

In another experiment, the applications were run on a single computer with the two convertors connected to its USB ports. All the communications between the applications took place as if two separate computers were used with no jitter problems detected. We have also conducted experiments with virtual COM ports created by the off-the-shelf com 2 com software package. In this case, no convertors were used and all software components were run on the same physical computer. We believe, therefore, that the development of the timecritical software components related to the sensor data communication can be simplified by running a unified SDK on a single, sufficiently powerful workstation.

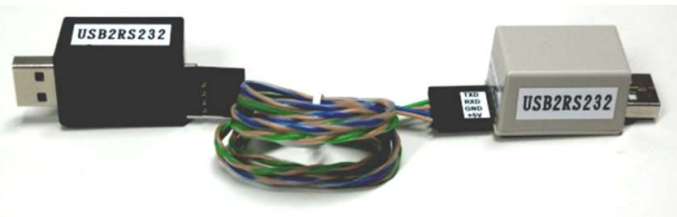

Figure 6. Two USB2RS232 convertors wired together. 
In the discussion so far, we have considered only the local use of the Data Glove which is confined to a single network segment (Intranet). While this is usually sufficient for development, laboratory experiments, and demos, in some cases, global access to the Data Glove becomes necessary. This happens, for example, when the Data Glove needs to be accessed from outside of the lab in which it is normally used in order to send realtime data to external parties. In this case, instead of physically coming to the lab, such external parties can videoconference for observing and giving instructions while monitoring the real-time feed from the Data Glove. We handle such situations, by tunneling the local server port of the Data Glove to a server, publicly accessible on the Internet by a secure shell connection.

Another scenario occurs when the Data Glove is used for demonstrations and experiments outside of the usual laboratory environment. In such cases, the standard network infrastructure that is configured to seamlessly integrate the Data Glove into the laboratory environment and provide access to it from different locally connected computers will not be accessible. To address this issue, we have acquired and configured the compact WiFi router shown in Figure 7 for the mobile use of the Data Glove.

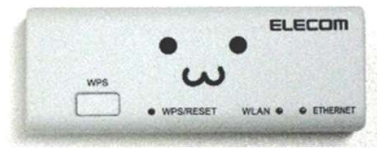

Figure 7 . The compact wireless router $(64 \times 18 \times 24 \mathrm{~mm})$ that was employed in mobile experiments with the Wireless Data Glove.

The Dynamic Host Configuration Protocol (DHCP) server of this router is configured to assign a predetermined fixed IP address to the Stick PC Data Glove Server based on its MAC address. At startup, the Stick PC automatically connects to the WiFi network created by the compact router and runs the Data Glove server software. In this way, the Data Glove becomes accessible for any other computer that is connected to the WiFi network of the mobile router. One may think that the Stick PC itself could be set up as a WiFi access point without the need to employ a router. However, our experiments, with this approach, have revealed that the current mobile hotspot functionality using Windows 10 is limited in the absence of an Internet connection. In contrast, the compact router approach that we have implemented does not require Internet access although the router can be easily connected to a Local Area Network (LAN) with Internet access, if necessary.

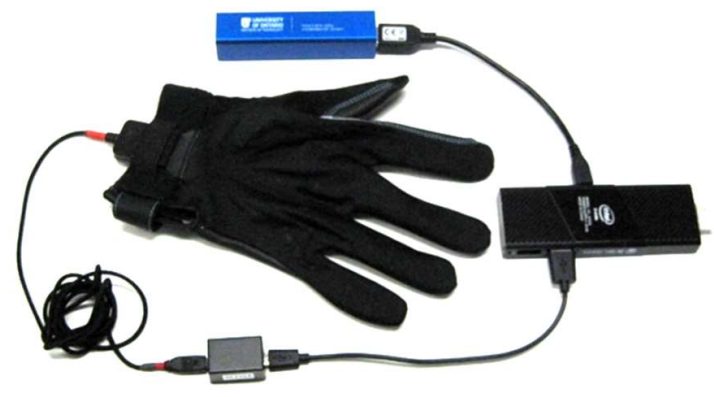

Figure 8. The wearable components of the wireless Data Glove set: the Data Glove in the middle, the USB2DG convertor in the bottom, the Stick PC on the right, and the mobile battery on the top.

By using the mobile Data Glove infrastructure described above, we have created a wearable version of the Data Glove. The components attached to the body of the user are shown in Figure 8. Note that no peripherals such as a keyboard, a mouse, or a screen are wired to the Stick PC. The only physical connections are to the USB2DG converter that is wired to the Data Glove, and the mobile battery.

Although the WiFi connection and the Data Glove server application are automatically initiated at startup, the Stick PC is also configured to accept Remote Desktop connection. In this way, the Stick PC and the Data Glove server application can be controlled remotely through the WiFi network of the compact router whenever necessary. An alternative approach for wireless control of the Stick PC would be to use a wireless display and Bluetooth keyboard and mouse.

\section{Conclusions and Future Works}

We have devised a CNT sensor production framework to encompass the research of novel highly stretchable CNT sensors and facilitate the fabrication of experimental components with special properties. Using the Yamaha Data Gloves that are based on an earlier version of our sensors, we have constructed a communication framework for testing and experimental work with different applications. For this, the USB2DG and USB2RS232 hardware convertors have been constructed and the ip2com and com2ip software communication components have been implemented. Wireless motion tracking experiments with the Yamaha Data Glove have been conducted to explore the autonomous data processing on a Stick PC and the remote data processing on a wirelessly connected workstation. A remote communications model with a client PC connected to a distant Data Glove server on the Internet was also explored. 
The Stick PC based wireless Data Glove approach provides a flexible solution that is indispensable for conducting experimental work. However, the deployment of Data Glove-based solutions that include both the necessary hardware and software prompts for more straightforward wireless solutions. Indeed, it will be easier for the end-users to have a dedicated wireless box that simply connects to the glove and works, rather than a fully-fledged Stick PC that needs continuous support and OS updates, etc. We are, therefore, experimenting with building the dedicated hardware for a Data Glove WiFi communication box that will require minimal, if any, maintenance.

Future work will see the employment of the Data Glove in real-world applications. More specifically, our ongoing work is focusing on the application of immersive technologies (virtual reality and virtual simulation, in particular), for medical education, with an emphasis on anesthesia training. However, given the issues/limitations associated with the currently available high-fidelity haptic solutions (e.g., cumbersome, cost prohibitive; see [29]), our work has focused primarily on cognitive skills (e.g., learning about the steps of a procedure, and the tools required at each step) development. The use of the Data Glove will enable us to overcome some of these limitations and implement more realistic virtual training simulations that allow for technical skills development in a cost-effective manner. Using the Data Glove within a virtual simulation for anesthesia training will allow users to naturally grab and hold objects the same way they would in a real setting. For example, consider the handling of a drug vial, common in anesthesia, where the medical professional will grasp the vial with their hand and "pop" the cap off with their thumb. Using the methods of interaction available with the current virtual reality systems (e.g., hand-held controllers), this is simulated with a button press, making it highly unrealistic. With the use of the Data Glove, we plan to support more natural interactions and thus allow the user to employ objects as they would in the real world.

Another line of work will deal with the development of an assistive hardware and software communication system in support of people with physical and cognitive impairments including deafblindness.

\section{Acknowledgment}

The research and development related to this work was partially supported by a Cooperative Research Project at the Research Center for Biomedical Engineering and the Research Institute of Electronics, Shizuoka University, Hamamatsu, Japan.

\section{References}

[1] D. J. Sturman and D. Zeltzer, "A survey of glove-based input," in IEEE Computer Graphics and Applications, Vol. 14, no. 1, pp. 30-39, Jan. 1994.

[2] L. Dipietro, A. M. Sabatini, and P. Dario, "A Survey of Glove-Based Systems and Their Applications," The IEEE Transactions on Systems, Man, and Cybernetics, Part C: Applications and Reviews, 2008. pp. 461-482.

[3] T. DeFanti and D. J. Sandin, "Sayre Glove Final Project Report," US NEA R60-34-163 Final Project Report, November 10, 1977.

[4] K. Kanev, M. De Marsico, and P. Bottoni, "A Human Computer Interactions Framework for Biometric User Identification," Jap. Journal of Applied Physics Conf. Proc., Vol. 4, 2016, pp.011601(1-6).

[5] K. Kanev, M. De Marsico, P/ Bottoni, and A. Mecca, "Mobiles and Wearables: Owner Biometrics and Authentication," AVI'16 Proceedings of the International Working Conference on Advanced Visual Interfaces, Bari, Italy, June 7-10, 2016, pp.318-319.

[6] M. Haghi, K. Thurow, and R. Stoll, "Wearable Devices in Medical Internet of Things: Scientific Research and Commercially Available Devices," Healthcare Informatics Research, Vol. 23. 2017, pp.. 4-15. 10.4258/hir.2017.23.1.4.

[7] P. Sarcevic, Z. Kincses, and S. Pletl, "Wireless Sensor Network based movement classification using wristmounted 9DOF sensor boards," Proceedings of the 2014 IEEE 15th International Symposium on Computational Intelligence and Informatics (CINTI), Budapest, 2014, pp. 85-90.

[8] L. A. F. Fernandes, V. F. Pamplona, J. L. Prauchner, L. P. Nedel, and M. M. Oliveira, "A Conceptual ImageBased Data Glove for Computer-Human Interaction," Revista de Informática Teórica e Aplicada. Vol.15, no.3, 2008, pp. 75-94.

[9] S. Furuya, A. Nakamura, and N. Nagata, "Acquisition of individuated finger movements through musical practice," Neuroscience, Vol. 275, 2014.

[10] J. MacRitchie and A. P. McPherson, "Integrating optical finger motion tracking with surface touch events," Front. Psychol., Vol. 6, no. 702. 2015.

[11] Y. D. Pra, F. Fontana, and L. Tao, "Infrared vs. Ultrasonic Finger Detection on a Virtual Piano Keyboard," Proceedings of the International Computer Music Association (ICMC), 2014.

[12] T. G. Zimmerman, "Optical Flex Sensor," US Patent 4 542 291, September 17, 1985.

[13] Y. L. Harvill, T. G. Zimmerman, and J. G. Grimaud, "Motion Sensor which Produces an Asymmetrical Signal in Response to Symmetrical Movement," US Patent 5097 252, March 17, 1992.

[14] O. Ivanov and A. Chertoriyskiy, "Fiber-Optic Bend Sensor Based on Double Cladding Fiber," Journal of Sensors, 2015, pp. 1-6.

[15] T. Zimmerman, J. Lanier, C. Blanchard, S. Bryson, and Y. Harvill, "A hand gesture interface device," $A C M$ Sigchi Bulletin, Vol. 17, 1986, pp. 189-192.

[16] J. S. Neely and P. J. Restle, "Capacitive Bend Sensor," US Patent 5610 528, March 11, 1977. 
[17] D. L. Gardner, "Inside story on: The power glove (Cover)" Design News, Vol. 45, no. 23:63, 4 December 1989.

[18] G. B. Langford, "Flexible Potentiometer," US Patent 5 583 476, December 10, 1996.

[19] I. Hirata, H. Nakamoto, H. Ootaka, and M. Tada, "The Flexible Interface Using a Stretch Sensor," Procedia Manufacturing, Vol. 3., 2015, pp. 845-849.

[20] A. Tairych and I. Anderson, "Capacitive Stretch Sensing for Robotic Skins," Soft Robotics, Vol. 6, 2019.

[21] O. Glauser, D. Panozzo, O. Hilliges, and O. SorkineHornung, "Deformation Capture via Soft and Stretchable Sensor Arrays," ACM Transactions on Graphics, Vol. 38, 2019, pp. 1-16.

[22] A. Firouzeh and J. Paik, "The Design and Modeling of a Novel Resistive Stretch Sensor With Tunable Sensitivity," IEEE Sensors Journal, Vol.15. 2015, pp. 6390-6398.

[23] L. Sbernini, A. Pallotti, and G. Saggio, "Evaluation of a Stretch Sensor for its inedited application in tracking hand finger movements," Proceedings of the IEEE International Symposium on Medical Measurements and Applications (MeMeA), Benevento, 2016, pp. 1-6.

[24] H. Lee, H. Cho, S. Kim, Y. Kim, and J. Kim, "Dispenser Printing of Piezo-resistive Nanocomposite on Woven Elastic Fabric and Hysteresis Compensation for SkinMountable Stretch Sensing," Smart Materials and Structures, Vol. 27, 2018.
[25] Y. Inoue, K. Kakihata, Y. Hirono, T. Horie, A. Ishida, and H. Mimura, "One-step grown aligned bulk carbon nanotubes by chloride mediated chemical vapor deposition," Applied Physics Letters, Vol. 92, 2008.

[26] Y. Inoue, Y. Suzuki, Y. Minami, J. Muramatsu, Y. Shimamura, K. Suzuki, A. Ghemes, M. Okada, S Sakakibara, H. Mimura, and K. Naito, "Anisotropic carbon nanotube papers fabricated from multiwalled carbon nanotube webs," Carbon, Vol. 49, 2011.

[27] K. Suzuki, K. Yataka, Y. Okumiya, S. Sakakibara, K. Sako, H. Mimura, and Y. Inoue, "Rapid-Response, Widely Stretchable Sensor of Aligned MWCNT/Elastomer Composites for Human Motion Detection," ACS Sensors, 2016

[28] M. Demoe, A. Uribe-Quevedo, A. Salgado, H. Mimura, K. Kanev, and P. C. K. Hung, "Exploring Data Glove and Robotic Hand Exergaming: Lessons Learned," Proceeding of the IEEE International Conference on Serious Games and Applications for Health (SeGAH) 2020, Vancouver, Canada, 2020.

[29] D. Panariello, T. Caporaso, S. Grazioso, G. Di Gironimo, A. Lanzotti, S. Knopp, and P. Klimant, "Using the KUKA LBR iiwa Robot as Haptic Device for Virtual Reality Training of Hip Replacement Surgery," Proceedigns of the 2019 Third IEEE International Conference on Robotic Computing (IRC), pp. 449-450 